\title{
Crises politico-militaires et dynamique de la végétation du Parc national du Mont Péko en Côte d’Ivoire
}

\section{Sidibé OUSMANE ${ }^{1}$ \\ Hyppolite N'DA DIBI ${ }^{1}$ \\ Kouadio Henri KouASSI ${ }^{2}$ \\ Konan Édouard KouASS ${ }^{1}$ \\ Kpolo OUATTARA ${ }^{3}$}

${ }^{1}$ Université Félix Houphouët-Boigny Unité de formation et de recherche en biosciences

23 BP 4957, Abidjan 23

Côte d'Ivoire

${ }^{2}$ Université Jean Lorougnon Guédé Unité de formation et de recherche en agroforesterie

BP 150, Daloa

Côte d'Ivoire

${ }^{3}$ Office ivoirien des parcs et réserves 06 BP 426, Abidjan 06

Côte d'Ivoire

\section{Auteur correspondant /}

Corresponding author:

Sidibé Ousmane -

sidibeousmane467@gmail.com

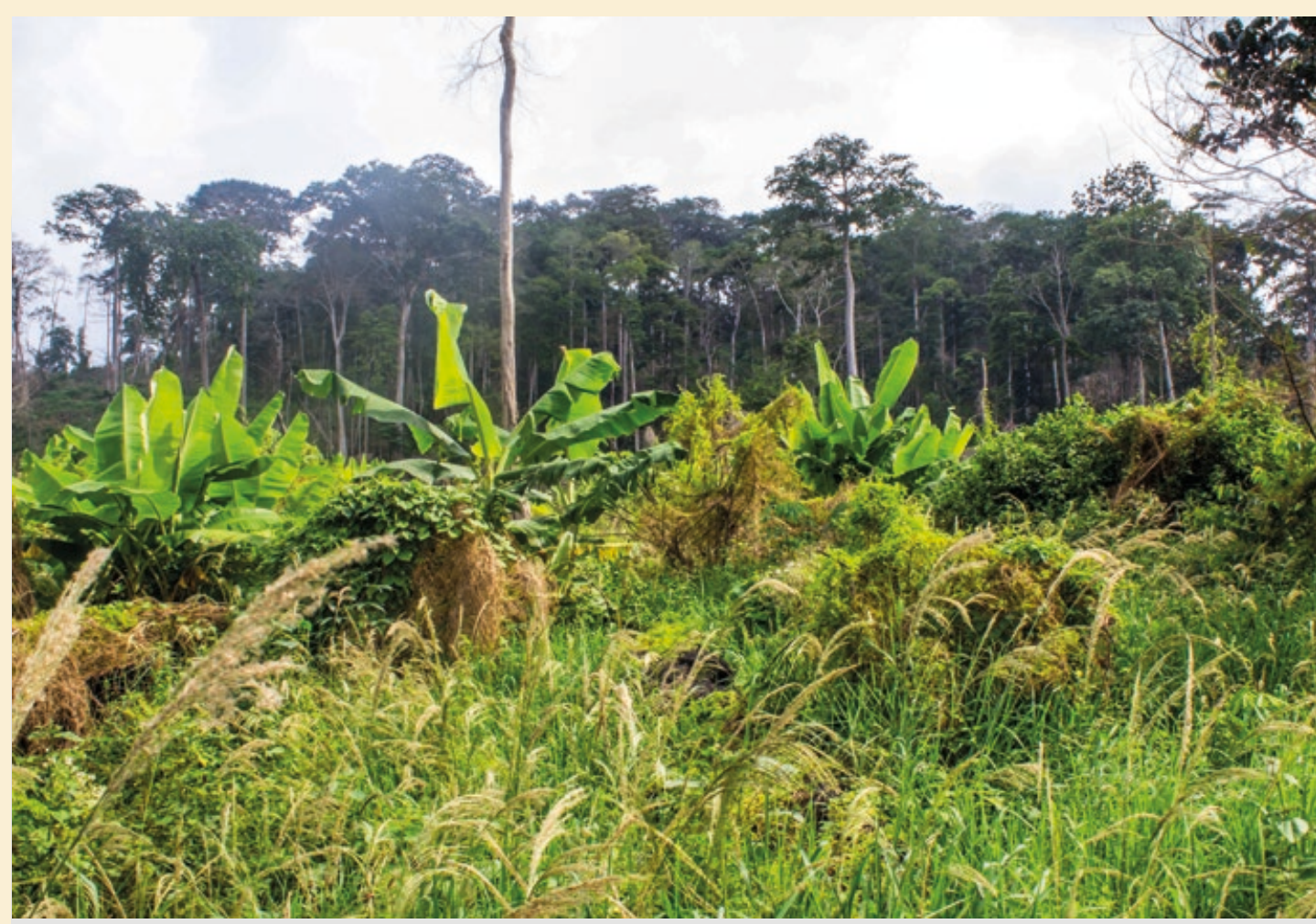

Photo 1.

Transformation de la forêt dense humide semi-décidue (en arrière-plan) en exploitation agricole (premier plan). Pendant et après la période des crises politico-militaires entre 2002 et 2011 , de vastes étendues de forêt ont été systématiquement éliminées au profit de la culture du cacao dans le Parc national du Mont Péko en Côte d'Ivoire.

Photo S. Ousmane.

Doi : 10.19182/bft2020.343.a31837 - Droit d'auteur (c) 2020, Bois et Forêts des Tropiques - (c) Cirad - Date de soumission : 19 avril 2019 ; date d'acceptation : 30 août 2019 ; date de publication : 2 janvier 2020.

\section{cirad (a)}

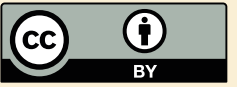

Licence Creative Commons :

Attribution - 4.0 International.

Attribution-4.0 International (CC BY 4.0)
Citer l'article / To cite the article

Ousmane S., N'da Dibi H., Kouassi K. H., Kouassi K. E., Ouattara K., 2020. Crises politico-militaires et dynamique de la végétation du Parc national du Mont Péko en Côte d'Ivoire. Bois et Forêts des Tropiques, 343 : 27-37. Doi : https://doi.org/10.19182/bft2020.343.a31837 


\section{RÉSUMÉ}

\section{Crises politico-militaires et dynamique de la végétation du Parc national du Mont Péko en Côte d'Ivoire}

Depuis sa création, le Parc national du Mont Péko est sujet à diverses pressions anthropiques. Ces pressions ont été accentuées durant les conflits armés en Côte d'Ivoire entre 2002 et 2011. L'intensification des pressions aurait entraîné une augmentation du taux de déforestation dans le parc. Pour vérifier cette hypothèse, la présente étude visait à déterminer l'effet des conflits sur la dynamique forestière du parc. À cet effet, quatre images satellitaires Landsat ont été analysées pour examiner les changements d'occupation des sols avant, pendant et après les conflits. Les résultats de ces travaux révèlent une forte réduction des surfaces forestières durant les conflits, qui s'est accentuée pendant la période post-conflit. En effet, 5,65 \% de pertes se sont produites avant les conflits à un taux annuel de 0,42\%, contre 28,03\% pendant les conflits à un taux annuel de $3,92 \%$, et $34,02 \%$ après les conflits à un taux annuel de 14,41\%. Cette aggravation de la déforestation dans le parc est liée à la culture illégale du cacao. L'infiltration massive des populations paysannes dans cette aire protégée pendant les conflits a entraîné une exacerbation des pressions. Une surveillance constante et un aménagement des zones converties en plantation de cacao afin d'accélérer la régénération dans le parc s'avèrent donc nécessaires. En outre, les résultats soulignent la vulnérabilité des aires protégées lors des conflits et l'urgence de la prise de mesures de sécurisation de celles-ci pour prévenir les risques de dégradation.

Mots-clés : conflits armés, déforestation, aire protégée, télédétection, Mont Péko, Côte d'Ivoire.

\section{ABSTRACT}

\section{Political-military crises and vegetation dynamics in Mont Péko National Park in Côte d'Ivoire}

Since its creation, Mont Péko National Park has been subject to various anthropogenic pressures, which increased during the period of armed conflict in Côte d'Ivoire between 2002 and 2011. The intensification of pressures appears to have brought an increased rate of deforestation in the park. To test this hypothesis, this study aimed to determine the effects of the conflict period on forest dynamics in the park. For this purpose, four Landsat satellite images were analysed to examine land use changes before, during and after the period of conflict. Our results show a sharp reduction in forested areas during the period of conflict, followed by further reductions in the post-conflict period. In percentage terms, $5.65 \%$ of losses occurred before the conflict period at an annual rate of $0.42 \%$, as against $28.03 \%$ during the conflicts at an annual rate of $3.92 \%$ and $34.02 \%$ after the conflicts at an annual rate of $14.41 \%$. This worsening deforestation in the park is linked to the illegal cultivation of cocoa. Existing pressures have thus been exacerbated by the largescale arrival of peasant populations in the protected area during the conflict period. Our results also show the vulnerability of protected areas during conflicts and the urgency of taking security measures to prevent the risk of degradation, thus further highlighting the need for constant monitoring and management to accelerate forest regeneration in park areas converted to cocoa plantations.

Keywords: armed conflict, deforestation, protected area, remote sensing, Mont Péko, Côte d'Ivoire.

\section{RESUMEN}

\section{Crisis politicomilitares y dinámica de la vegetación del Parque Nacional del Monte Péko en Costa de Marfil}

Desde su creación, el Parque Nacional del Monte Péko ha sufrido varias presiones antrópicas. Estas presiones se acentuaron durante los conflictos armados que tuvieron lugar en Costa de Marfil entre 2002 y 2011. La intensificación de las presiones podría haber comportado un aumento de la tasa de deforestación en el parque. Para verificar esta hipótesis, el presente estudio pretende determinar el efecto de los conflictos en la dinámica forestal del parque. Con este objetivo, se analizaron cuatro imágenes de satélite Landsat para examinar los cambios de ocupación de los suelos antes, durante y después de los conflictos. Los resultados de estas investigaciones revelan una importante reducción de las superficies forestales durante los conflictos, lo que se acentuó en el período postconflicto. Efectivamente, el 5,65\% de las pérdidas se produjeron antes de los conflictos con una tasa anual del 0,42 \%, ante el $28,03 \%$ durante los conflictos con una tasa anual del 3,92 \%, y del 34,02 \% después de los conflictos con una tasa anual del 14,41 \%. Esta agravación de la deforestación en el parque está relacionada con el cultivo ilegal de cacao. La infiltración masiva de poblaciones rurales en la zona protegida durante los conflictos comportó una intensificación de las presiones. Por ello, resulta necesaria una vigilancia constante y una gestión de las zonas convertidas en plantación de cacao para acelerar la regeneración en el parque. Además, los resultados destacan la vulnerabilidad de las zonas protegidas durante los conflictos y la urgencia de la toma de medidas de seguridad para prevenir los riesgos de degradación.

Palabras clave: conflictos armados, deforestación, zona protegida, teledetección, Monte Péko, Costa de Marfil 


\section{Introduction}

De la fin de la Seconde Guerre mondiale à 2017, le monde a connu 285 conflits armés engageant au moins le gouvernement d'un État dans 157 pays (Pettersson et Eck, 2018). Ces conflits ont des conséquences au plan social, politique et environnemental (Nackoney et al., 2014). Au plan environnemental, les impacts des conflits relèvent de trois processus distincts (Mc Neely, 2003). En premier lieu, les conflits peuvent conduire à une diminution des pressions anthropiques sur les ressources naturelles dans les zones de combat et de non-belligérance (Kim, 1997 ; Alvarez, 2003). En deuxième lieu, ces conflits peuvent entraîner une utilisation accrue ou inefficace des ressources naturelles, telle que la consommation de bois d'œuvre et de chauffage à proximité des camps de réfugiés (Gorsevski et al., 2013), le défrichement des forêts (Alix-Garcia et al., 2013), la croissance urbaine (Alix-Garcia et Saah, 2010) et le braconnage incontrôlé par les réfugiés et les combattants (Dudley et al., 2002). En dernier lieu, un conflit armé peut causer l'effondrement des institutions étatiques en charge de la protection de l'environnement (Mc Neely, 2003 ; Allnutt et al., 2013). Cette situation entraîne des activités illicites de l'homme dans les aires protégées, notamment l'exploitation forestière et minière illégale, l'agriculture (photo 1) et le braconnage (Allnutt et al., 2013 ; Barima et al., 2016).

En Côte d'Ivoire, les crises politico-militaires déclenchées en septembre 2002 ont abouti à la division du pays en trois parties. La partie Sud est restée sous contrôle gouvernemental, la partie Nord est passée sous contrôle d'une rébellion armée, et à l'intersection de ces deux parties une « zone tampon » de non-belligérance a été délimitée. Cette partie était placée sous le contrôle des forces onusiennes et françaises. Outre les répercussions sociales, ces crises ont impacté l'environnement (PNUE, 2015). Les impacts environnementaux ont été plus accentués dans les zones forestières de la zone tampon (Bamba et al., 2018), où la culture de cacao a été à la base d'une forte déforestation (Kouakou et al., 2015 ; Assalé et al., 2016 ; Barima et al., 2016). Malheureusement, les formations forestières des forêts classées et des aires protégées situées dans la zone tampon n'ont pas été épargnées. Le Parc national du Mont Péko (PNMP) en est une parfaite illustration. En effet, soustrait du contrôle de l'État, du fait de sa localisation dans la zone tampon, cette aire protégée a été occupée par une bande armée. Cette dernière y a induit la création de campements, la mise en place de vastes plantations de cacao et l'exploitation forestière illégale (OIPR, 2017). Malgré la fin des conflits en 2011, le parc est resté illégalement occupé et exploité par les populations jusqu'en 2016.

L'Office ivoirien des parcs et réserves (OIPR), après avoir démantelé la bande armée qui contrôlait le parc en 2013, puis achevé l'opération d'exfiltration des occupants illégaux en 2016, s'est engagé dans un processus de réhabilitation du PNMP. Il se pose alors beaucoup de questions auxquelles il faudrait répondre pour réussir la réhabilitation du parc: Quelle a été la contribution réelle de la crise dans l'accélération de la déforestation dans le PNMP?

Quelles sont les ressources forestières encore intactes dans le parc ? Comment se répartissent les exploitations agricoles clandestines dans le parc ? C'est dans ce contexte que cette étude visant à déterminer l'effet des conflits armés sur la dynamique forestière du parc a été réalisée afin de proposer des stratégies de réhabilitation. Ce travail repose sur l'hypothèse que l'anthropisation du parc pendant et après les conflits armés a accentué la déforestation. De façon spécifique, il s'agissait de produire les données sur les ressources forestières du PNMP, d'analyser les transformations de celles-ci avant (1988-2002), pendant (2002-2011) et après (2011-2016) les conflits, puis d'évaluer l'impact des conflits sur la déforestation.

\section{Méthodes}

\section{Milieu d'étude}

Le PNMP est à cheval sur les départements de Bangolo et de Duékoué dans la région du Guémon, à l'ouest de la Côte d'Ivoire. Située entre $6^{\circ} 53^{\prime}$ et $7^{\circ} 08^{\prime}$ de latitude Nord et $7^{\circ} 11^{\prime}$ et $7^{\circ} 21^{\prime}$ de longitude Ouest, cette aire protégée a échappé au contrôle de l'État pendant la période des crises politico-militaires (figure 1). Ces crises ont amplifié diverses pressions anthropiques sur cette aire protégée (Ousmane et al., 2018), notamment l'agriculture et l'exploitation forestière (photos 2 et 3 ).

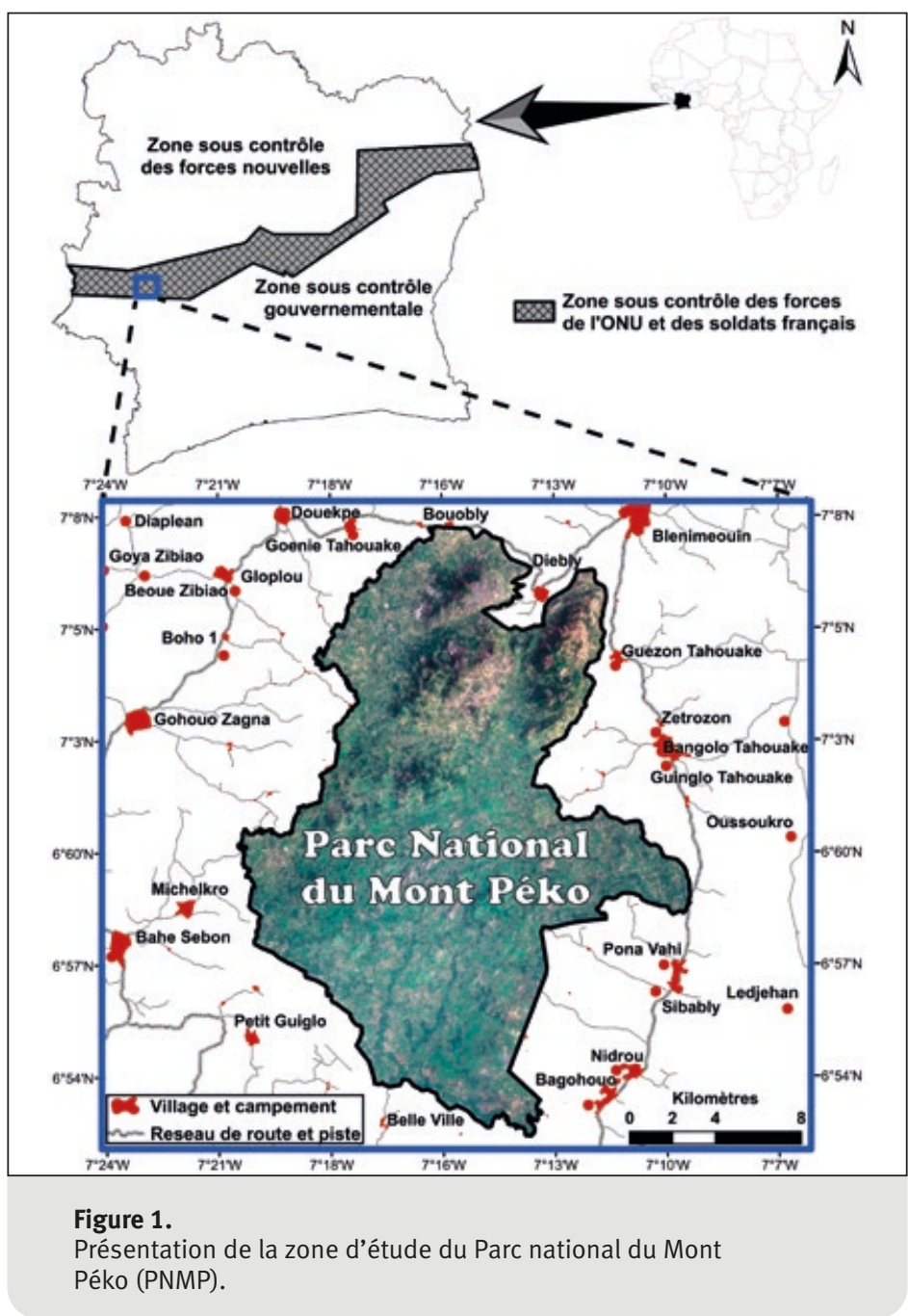




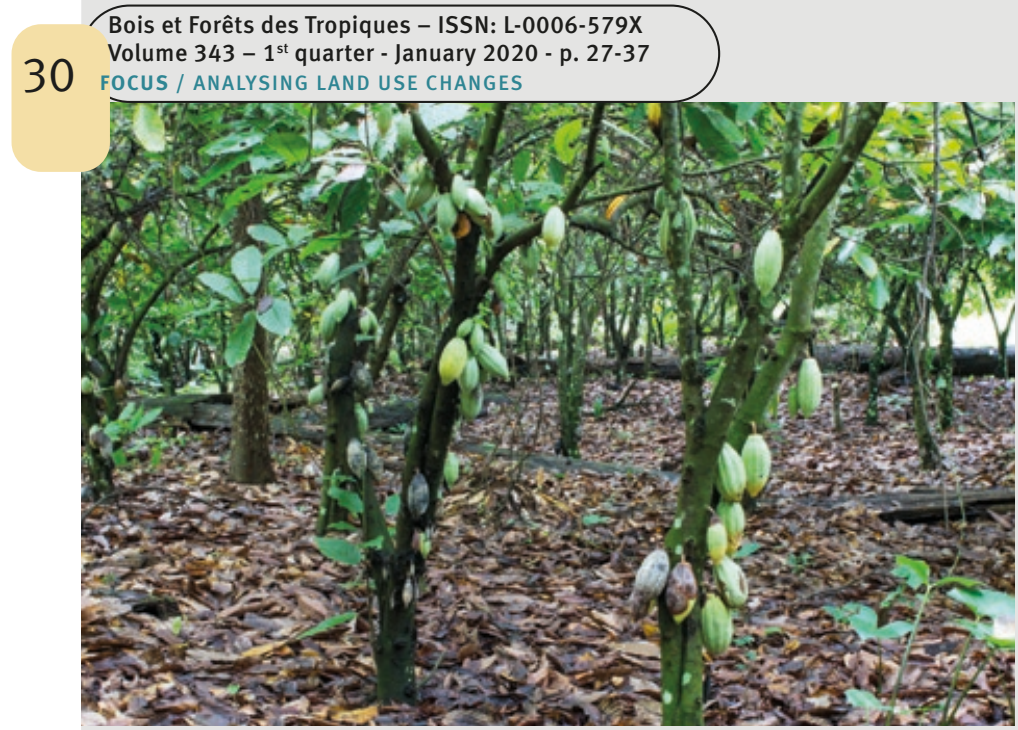

Photo 2.

Aperçu d'une plantation de cacao installée au cœur du Parc national du Mont Péko durant la période des conflits entre 2002 et 2011 en Côte d'Ivoire.

Photo S. Ousmane.

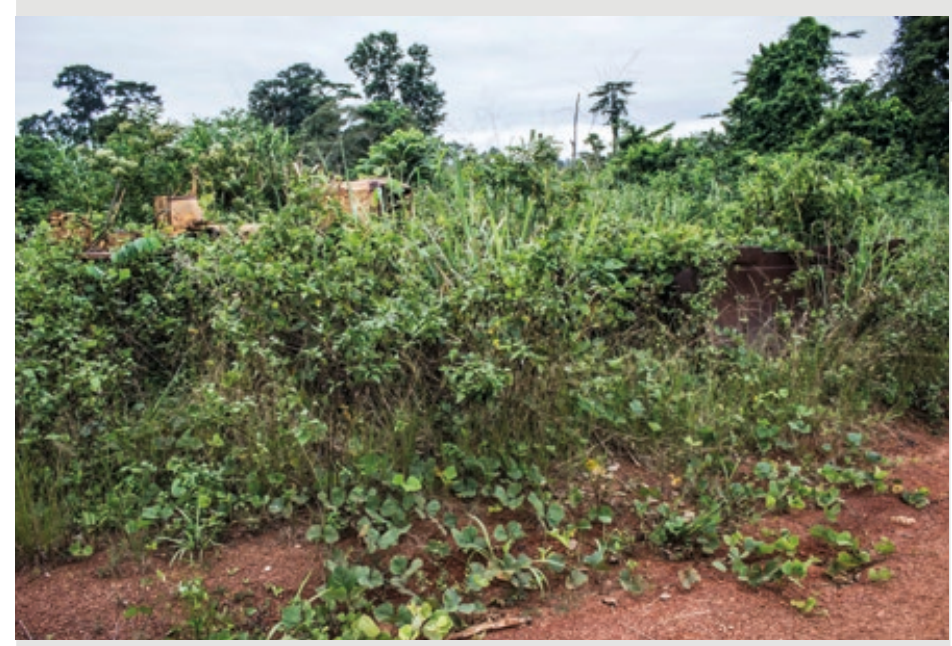

Photo 3.

Bulldozer abandonné par les exploitants forestiers à la fin des crises, à proximité du Parc national du Mont Péko, dans la localité de Michelkro au sud-ouest du parc. Photo S. Ousmane.

Le décret de création n 68-79 du 9 février 1968 luì confère une superficie de 34000 ha qui s'avère inférieure sur le terrain, en raison de la délimitation erronée du parc en 1974 (Ousmane et al., 2018). Le relief du parc est essentiellement caractérisé par des plateaux à peine vallonnés de 300 à $500 \mathrm{~m}$ d'altitude et trois sommets bien individualisés dans la partie Nord. Il s'agit des monts Péko (1 010 m), Kahoué (1 115 m) et Guéhi (918 m ; Avenard, 1971). Le climat de la région est de type subéquatorial humide à deux saisons, dont la pluvieuse s'étend de mars à octobre et la sèche de novembre à février (Eldin, 1971). La végétation est constituée de forêt humide semi-décidue, de forêt dégradée et de vastes exploitations agricoles résultant des activités humaines (UICN, 1996 ; OIPR, 2017). Appartenant au Guinean Forests of West Africa Hotspot, l'une des 18 zones tropicales d'importance mondiale pour leur biodiversité (CEPF,

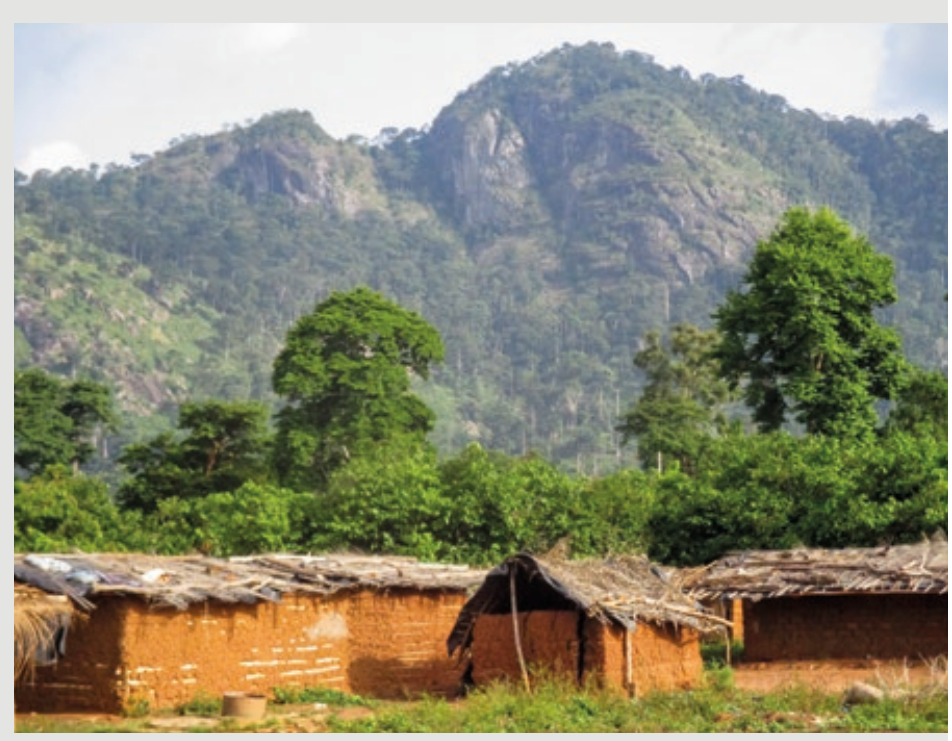

Photo 4.

Illustration de la pression foncière exercée sur le Parc national du Mont Péko montrant une implantation de village (Bouobly) dans la périphérie du parc.

Photo S. Ousmane.

2000), le parc héberge des espèces fauniques et floristiques à haute valeur de conservation telles que Chrysophyllum azaguieanum J. Miège, une plante en danger, Picarthates gymnocephalus Lesson 1828, une espèce d'oiseau rare, et deux grands mammifères en danger : Pan troglodytes verus Blumenbach 1776, le chimpanzé commun, et Loxodonta cyclotis Matschie 1900, l'éléphant de forêt (Lauginie et al., 2007 ; Goné Bi et al., 2013).

La zone périphérique du PNMP comprend une vingtaine de villages appartenant à trois grands cantons Guéré que sont les cantons Zagna, Tahouaké et Zagné. Du fait de la démographie galopante et de la raréfaction des terres arables dans la zone, ces villages exercent une pression foncière liée à l'étalement des villages et aux activités agricoles à la périphérie du parc (photo 4). Cette pression est le résultat de la confusion créée par le tracé erroné, en 1974, de la limite du décret de création, communément appelée limite Goué (figure 2).

\section{Données}

La réalisation de ce travail a nécessité l'utilisation de quatre images satellitaires Landsat, téléchargées sur le site web de l'United States Geological Survey ${ }^{1}$. Ces images couvraient les périodes d'avant, pendant et après les crises (tableau I).

Un GPS (Global Positionning System) Garmin GPSMAP 62s a été utilisé en 2016 par l'équipe de recherche au cours des différentes missions de prospection dans le parc pour l'enregistrement de points géolocalisés. Ces points GPS ont été utilisés pour réaliser et valider la classification des images satellitaires. Le logiciel ENVI 5.0 a été retenu pour le traitement des images satellitaires ainsi que ArcGIS.10.0 pour les analyses et les restitutions cartographiques.

1 United States Geological Survey (USGS), organisation gouvernementale américaine de prospection géologique : https://earthexplorer.usgs.gov 


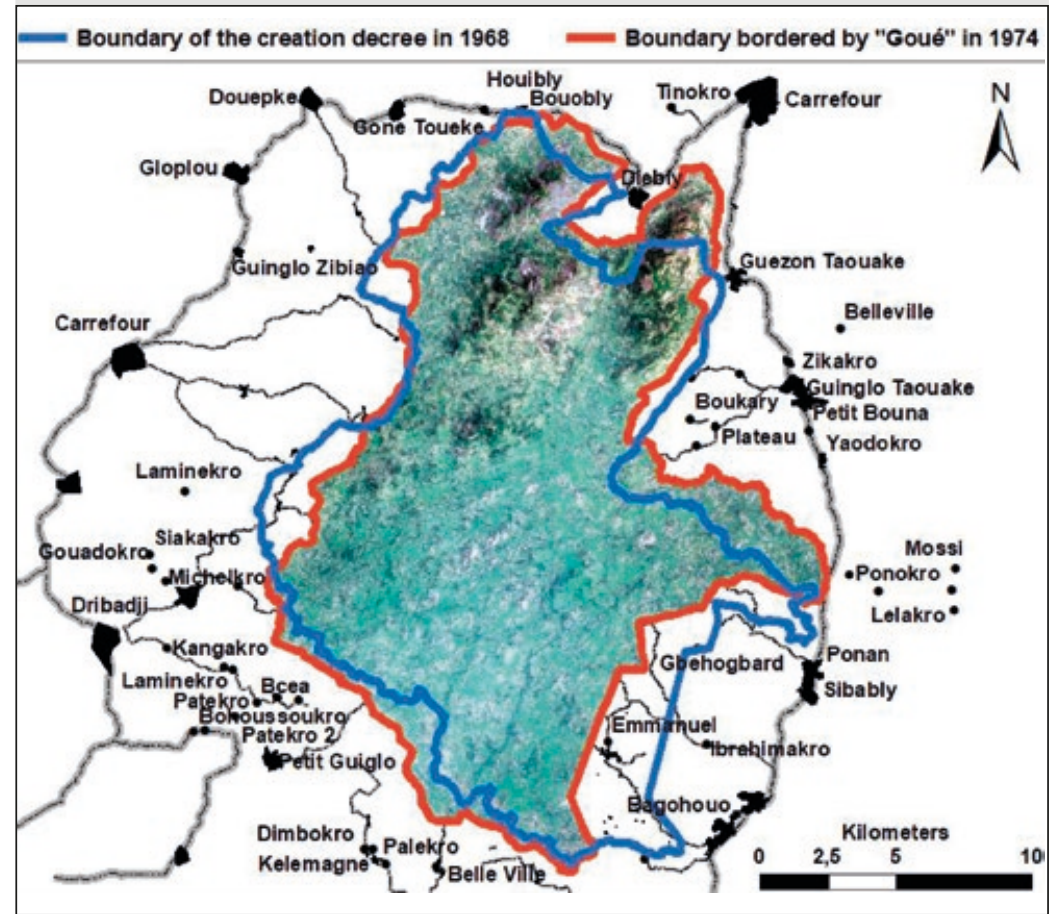

Figure 2.

Illustration de la confusion des limites du Parc national du Mont Péko (PNMP) (Ousmane et al., 2018).

\section{Cartographie de l'occupation des sols}

Les images acquises ont d'abord subi une calibration radiométrique et atmosphérique à l'aide de l'outil FLAASH (Fast Line of Sight Atmospheric Analysis of Spectral Hypercubes) implémenté dans le module Atmospheric correction du logiciel ENVI. Étant donné que toutes les images acquises avaient un géoréférencement correct, l'opération suivante a consisté à extraire les zones d'étude des images satellitaires à partir d'un fichier forme (shapfile) mis à disposition par l'OIPR. Compte tenu des objectifs de cette étude, une typologie simplifiée de l'occupation des sols a été adoptée. Il s'est agi des classes forêt, non-forêt et sols nus et affleurements rocheux. Les compositions colorées ont été réalisées à partir des bandes spectrales proche infrarouge, moyen infrarouge et rouge $(5 / 6 / 4)$ de l'image Landsat OLI de 2016 et (4/5/3) des images Landsat TM de 1988, 2011 et ETM de 2002. Ces compositions colorées ont permis de caractériser les formations forestières en rouge sombre, les sols nus et affleurements rocheux en cyan, et les non-forêts (cultures et jachères) dans une variante de couleur allant du rouge clair au vert en passant par le magenta.
Sur la base de cette interprétation visuelle, un total de 200 points (75 pour les forêts, 105 pour les non-forêts et 20 pour les sols nus et affleurements rocheux) ont été sélectionnés et leurs coordonnées géographiques enregistrées dans un GPS. Ces points nous ont guidés lors de nos missions de terrain et ont permis d'établir une correspondance entre coloration sur l'image fausse couleur et types d'occupation des sols. Pour évaluer l'accessibilité de ces sites, le réseau de pistes du site d'étude a été superposé aux images, puis un entretien avec les agents de l'OIPR a permis de valider les points sélectionnés.

Au cours des missions, 491 nouveauxpoints devant servir à la validation des traitements ont été enregistrés tous azimuts sur le terrain, dans les différentes classes d'occupation des sols (140 pour les forêts, 198 pour les non-forêts et 153 pour les sols nus et affleurements rocheux). De retour de terrain, les 200 points présélectionnés et effectivement visités ont servi de base à la sélection de parcelles d'entraînement pour la classification de l'image Landsat de 2016. Quant aux images des années antérieures, les points (occupation des sols) restés invariants jusqu'en 2016 ont été utilisés comme repères dans la sélection des parcelles d'entraînement. Pour la validation de la carte de 2016, la totalité des 491 points collectés ont servi de base à la digitalisation de parcelles de contrôle. Par contre, au niveau des cartes historiques (1998, 2002 et 2011), seuls les points de contrôle restés invariants jusqu'en 2016 ont servi de base à l'élaboration des matrices de confusion. Le point invariant est ici défini comme un site qui a gardé le même type d'occupation des sols pendant toute la période d'étude. Aussi, la sélection des sites invariants s'est appuyée sur la signature spectrale de l'occupation des sols, les connaissances de terrain, les images d'archive Google Earth de la zone remontant à 1984 et, enfin, sur les avis des gestionnaires du parc (les agents de l'OIPR).

Les performances des classifications ont été analysées par les indices de la précision globale et le coefficient de Kappa issus de la matrice de confusion (Girard et Girard, 1999). Pour la précision globale, un seuil minimum de $80 \%$ était visé.

Après la validation des images, les pixels isolés ont été éliminés par l'application d'un filtre médian $3 \times 3$.

Tableau I.

Caractéristiques des images satellitaires Landsat utilisées dans cette étude. TM = Thematic Mapper ; $\mathrm{ETM}=$ Enhance Thematic Mapper; OLI = The Operational Land Imager $;$ TIRS = Thermal Infrared Sensor.

\begin{tabular}{l|l|l|l|l|}
\hline No & Dates & Identifiants de la scène & Capteur & Résolution (m) \\
\hline 1 & $14 / 12 / 1988$ & LT51980551988349MPS00 & Landsat 5 TM & 30 \\
\hline 2 & $13 / 12 / 2002$ & LE71980552002347EDC00 & Landsat 7 ETM & 30 \\
\hline 3 & $19 / 12 / 2011$ & LC81980552013353LGN01 & Landsat 5 TM & 30 \\
\hline 4 & $27 / 12 / 2016$ & LC81980552016362LGN00 & Landsat 8 OLI TIRS & 30 \\
\hline
\end{tabular}




\section{Analyse de la dynamique forestière}

Les changements d'occupation des sols intervenus ont été mis en évidence à l'aide d'une matrice de transition (Schlaepfer, 2002). Les superficies des différentes classes d'occupation des sols ont été calculées à partir du croisement des différentes cartes d'occupation des sols à l'aide de la fonction Thematic change de la boîte à outils (Toolbox) du logiciel ENVI 5.0. Puis, le taux de changement (TC) global de chacune des classes d'occupation des sols au cours des différentes périodes étudiées a été déterminé suivant l'équation proposée par la FAO (1990) :

$T C=\frac{\left(S_{2}-S_{1}\right)}{S_{1}} \times 100$ (équation 1$)$

avec $S_{1}$ : superficie à la date $1 ; S_{2}$ : superficie à la date 2 .

Les différents taux de changement (TC) globaux ont été normalisés à partir du taux de changement initial de la classe forêt (1988-2002), afin de pallier les différences de poids entre les valeurs inter-périodes. La formule de normalisation était la suivante :

$T c_{y}=\frac{\left(P c_{y} \times T c_{x}\right)}{P c_{x}}$ (équation 2)

avec $T c_{x}$ et $P c_{x}$ respectivement, le taux de changement (\%) et la proportion de changement $\left(\mathrm{km}^{2}\right)$ de la classe forêt durant la période initiale étudiée; $P c_{y}$ la proportion de changement $\left(\mathrm{km}^{2}\right)$ de la période.

Enfin, le taux de déforestation annuel ( $r$ ) a été déterminé pour chaque période étudiée suivant la formule standardisée proposée par Puyravaud (2003) :

$r=\left(\left(\frac{1}{t_{2}-t_{1}}\right) \times \ln \left(\frac{S_{2}}{S_{1}}\right)\right) \times 100$ (équation 3)

Dans cette équation, $r$ représente le taux de variation annuel en pourcentage pour chaque classe d'occupation des sols et $S_{1}$ et $S_{2}$ sont la superficie de chaque classe à la date $t_{1}$ et $t_{2}$. Les taux négatifs représentent des pertes de forêt et les taux positifs des gains de forêt.

Ces différents calculs ont été effectués puis comparés sur les trois périodes suivantes : avant les conflits (19882002) ; pendant les conflits (2002-2011) ; après les conflits (2011-2016).

Tableau II.

Indices de performance des classifications.

\begin{tabular}{|l|r|r|r|r|}
\hline Indices & $\mathbf{1 9 8 8}$ & $\mathbf{2 0 0 2}$ & $\mathbf{2 0 1 1}$ & $\mathbf{2 0 1 6}$ \\
\hline & & & & \\
\hline Précision globale & 97,60 & 94,32 & 90,17 & 89,38 \\
\hline Coefficient Kappa & 0,91 & 0,86 & 0,82 & 0,83 \\
\hline
\end{tabular}

016

, 38
1988

2002
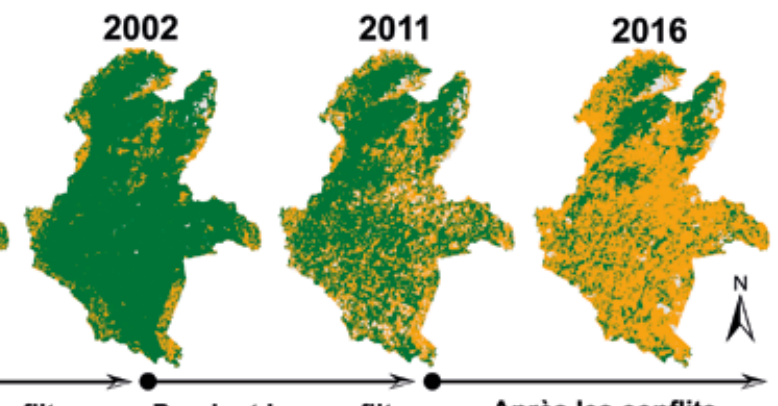

Avant les conflits

Pendant les conflits

Après les conflits

Forêt

\section{Non Forêt}

(Culture / Jachère)

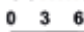

Kilomètres
Figure 3.

Cartes d'occupation des sols du Parc national du Mont Péko (PNMP) avant (1988-2002), pendant (2002-2011), et après (2011-2016) les conflits armés en Côte d'Ivoire.

\section{Résultats}

\section{Précision des classifications et cartographie des types d'occupation des sols}

La matrice de confusion des images Landsat classifiées donne des valeurs comprises entre $89,38 \%-97,60 \%$ et 0,82 - 0,91 respectivement pour la précision globale et le coefficient de Kappa (tableau II). Ces valeurs indiquent une bonne discrimination des différentes classes d'occupation des sols (Pontius, 2000). La figure 3 présente les cartes obtenues après les classifications supervisées des quatre images Landsat couvrant les périodes d'avant, pendant et après les crises. Au total, trois classes d'occupation des sols ont été cartographiées. Il s'agit des forêts (primaires ou secondaires), des non-forêts (cultures et jachères) et des sols nus et affleurements rocheux. L'analyse visuelle des cartes a révélé une forte modification des différents types d'occupation des sols entre 2002-2011 (pendant les conflits) et 2011-2016 (après les conflits).

\section{Dynamique de l'occupation des sols}

Le tableau III présente la composition de l'occupation sol du PNMP en 1988, 2002, 2011 et 2016. Les tendances générales observées sont une régression considérable des superficies forestières, une expansion des surfaces occupées par la classe des non-forêts (cultures et jachères) et une légère augmentation de superficie des sols nus et affleurements rocheux (figure 4). Avant les conflits (19882002), la couverture forestière a régressé. Elle est passée de $286,23 \mathrm{~km}^{2}(94,63 \%)$ à $270,05 \mathrm{~km}^{2}(89,28 \%)$, avec un taux de réduction de 5,65\%. Également, la classe des sols nus et affleurements rocheux a faiblement régressé $(-0,05 \%)$. En revanche, la classe des non-forêts a enregistré un taux d'augmentation de 5,70 \%. Pendant les conflits (20022011), la déforestation dans le PNMP s'est accentuée. Les surfaces forestières sont passées de $270,05 \mathrm{~km}^{2}(89,28 \%)$ au début des crises en 2002 à 189,77 km² (62,74\%) à la fin des crises en 2011 , soit un taux de perte de 28,03\%. 
Tableau III.

Superficie des classes d'occupation des sols du Parc national du Mont Péko en 1988, 2002, 2011 et 2016.

\begin{tabular}{|c|c|c|c|c|c|c|c|c|}
\hline \multirow{2}{*}{ Types d'occupation du sol } & \multicolumn{2}{|c|}{1988} & \multicolumn{2}{|c|}{2002} & \multicolumn{2}{|c|}{2011} & \multicolumn{2}{|c|}{2016} \\
\hline & $\mathrm{km}^{2}$ & $\%$ & $\mathrm{~km}^{2}$ & $\%$ & $\mathrm{~km}^{2}$ & $\%$ & $\mathrm{~km}^{2}$ & $\%$ \\
\hline Forêts & 286,23 & 94,63 & 270,05 & 89,28 & 189,77 & 62,74 & 92,32 & 30,52 \\
\hline Non-forêts (cultures et jachères) & 12,80 & 4,23 & 29,13 & 9,63 & 94,71 & 31,31 & 201,78 & 66,71 \\
\hline Sols nus / affleurements rocheux & 3,45 & 1,14 & 3,30 & 1,09 & 18,00 & 5,95 & 8,39 & 2,77 \\
\hline Total & 302,49 & 100 & 302,49 & 100 & 302,49 & 100 & 302,49 & 100 \\
\hline
\end{tabular}

L'on note cependant un taux d'augmentation élevé de la classe des non-forêts $(22,90 \%)$. De même, la classe des sols nus et affleurements rocheux a connu des gains en superficie (5,13\%). Après les crises (2011-2016), la tendance de déforestation déjà alarmante du PNMP s'est accrue. Le taux de perte forestière le plus élevé a été enregistré à cette période $\left(-34,04 \%\right.$, soit $\left.97,45 \mathrm{~km}^{2}\right)$. La classe des sols nus et affleurements rocheux a connu une régression de superficie de l'ordre de 3,36 \%. Cependant, la classe des non-forêts a connu sa plus forte augmentation $\left(37,38 \%\right.$, soit $\left.107,01 \mathrm{~km}^{2}\right)$. En 2016 , avec une superficie de $201,78 \mathrm{~km}^{2}$ $(66,71 \%)$, la classe des non-forêts (cultures et jachères) domine largement la matrice paysagère du PNMP.

Globalement, la matrice de transi-

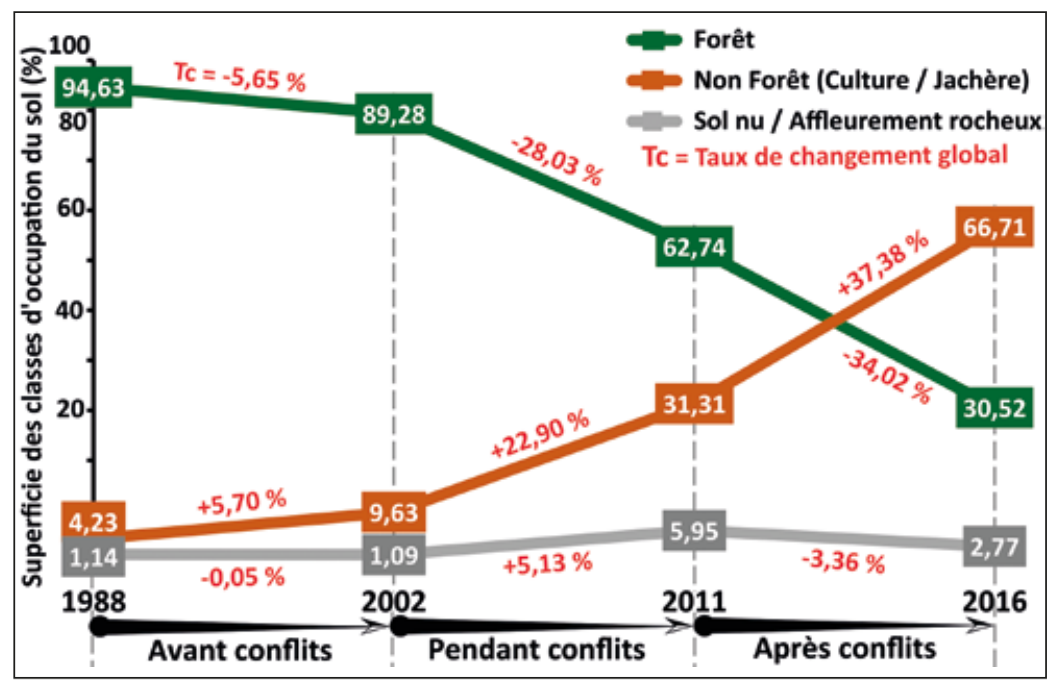

Figure 4.

Évolution de la superficie des différentes classes d'occupation des sols du Parc national du Mont Péko (PNMP), avant, pendant et après les conflits armés de 2002 à 2011.

tion (tableau IV) met en évidence un phé-

nomène de conversion des surfaces forestières du PNMP en non-forêts au cours des différentes périodes étudiées. Au cours de la période précédant les conflits, le flux était faible, à raison de 5,25\% sur 14 ans. Durant la décennie des conflits, la proportion de surfaces forestières converties en non-forêts (cultures et jachères) a fortement augmenté (23,05\%). À la sortie de la crise post-électorale de 2011 à 2016, le phénomène de déforestation s'est intensifié dans le PNMP. En seulement 5 ans, 31,81 \% de forêts ont été converties en cultures et jachères.

En somme, le PNMP a subi une forte déforestation entre 1988 et 2016 (tableau V). Toutefois, cette déforestation était moins contrastée avant les conflits. C'est pendant et après les conflits que les proportions de pertes forestières ont fortement augmenté, à raison de 80,28 km² $^{2}$ entre 2002-2011 et $97,45 \mathrm{~km}^{2}$ entre $2011-2016$. Avec une perte de $14,41 \%$ par an, le taux de déforestation annuel le plus élevé a été observé après les conflits.

\section{Discussion}

La présente étude a révélé une déforestation dans le PNMP entre 1988 et 2016 . Les proportions de pertes forestières enregistrées ont été très élevées pendant et après les conflits. Ces résultats corroborent ceux de Barima et al. (2016) au sein de la forêt classée du Haut-Sassandra (FCSH) dans le département de Daloa. Comparativement aux résultats des travaux de Ordway (2015) sur la réserve forestière de Gishwati et de Mukura, dans la région d'Afromontane, nos résultats ont révélé une déforestation beaucoup plus prononcée dans le PNMP. Globalement, l'analyse des matrices de transition de la composition du paysage au cours des périodes étudiées indique une conversion des zones forestières en non-forêts (cultures et jachères). Plusieurs auteurs ont fait ce constat dans des études de détection de changement d'occupation des sols, sur des périodes approximativement équivalentes à celles de la présente étude (Kouakou et al., 2015 ; Bamba et al., 2018). 
Tableau IV.

Matrice de transition de l'occupation des sols dans le Parc national du Mont Péko entre 1988 et 2016.

\begin{tabular}{|c|c|c|c|c|c|}
\hline & $\begin{array}{l}\text { Types d'occupation } \\
\text { des sols (\%) }\end{array}$ & Forêts & $\begin{array}{l}\text { Non-forêts } \\
\text { (cultures } \\
\text { et jachères) }\end{array}$ & $\begin{array}{c}\text { Sols nus / } \\
\text { affleurements } \\
\text { rocheux }\end{array}$ & Total \\
\hline \multirow{5}{*}{1988} & & 2002 & & & \\
\hline & Forêts & 89,26 & 5,25 & 0,12 & 94,63 \\
\hline & Non-forêts (cultures et jachères) & 0,02 & 4,19 & 0,03 & 4,23 \\
\hline & Sols nus / affleurements rocheux & 0,00 & 0,20 & 0,94 & 1,14 \\
\hline & Total & 89,28 & 9,63 & 1,09 & 100 \\
\hline \multirow{5}{*}{2002} & & 2011 & & & \\
\hline & Forêts & 62,74 & 23,05 & 3,49 & 89,28 \\
\hline & Non-forêts (cultures et jachères) & 0,00 & 8,01 & 1,62 & 9,63 \\
\hline & Sols nus / affleurements rocheux & 0,00 & 0,25 & 0,84 & 1,09 \\
\hline & Total & 62,74 & 31,31 & 5,95 & 100 \\
\hline \multirow{5}{*}{2011} & & 2016 & & & \\
\hline & Forêts & 30,52 & 31,81 & 0,40 & 62,74 \\
\hline & Non-forêts (cultures et jachères) & 0,00 & 30,23 & 1,08 & 31,31 \\
\hline & Sols nus / affleurements rocheux & 0,00 & 4,67 & 1,29 & 5,95 \\
\hline & Total & 30,52 & 66,71 & 2,77 & 100 \\
\hline
\end{tabular}

\begin{tabular}{|c|c|c|}
\hline \multicolumn{3}{|c|}{$\begin{array}{l}\text { Tableau V. } \\
\text { Évolution de la déforestation dans le PNMP } \\
\text { entre } 1988 \text { et } 2016 .\end{array}$} \\
\hline \multirow[t]{2}{*}{ Période } & \multicolumn{2}{|c|}{ Déforestation } \\
\hline & $\begin{array}{l}\text { Total } \\
\left(\mathrm{km}^{2}\right)\end{array}$ & $\begin{array}{c}\text { Taux annuel } \\
\text { r (\%) }\end{array}$ \\
\hline Avant les conflits (1988-2002) & 16,18 & $-0,42$ \\
\hline Pendant les conflits (2002-2011) & 80,28 & $-3,92$ \\
\hline Après les conflits (2011-2016) & 97,45 & $-14,41$ \\
\hline
\end{tabular}

Avec 4,28\% de superficies des non-forêts dans le PNMP en 1988, il ressort que l'infiltration des agriculteurs dans le PNMP a commencé avant le début des conflits en Côte d'Ivoire en 2002. À cette période, les non-forêts (cultures et jachères) étaient généralement situées à la périphérie du parc adjacente à la zone rurale. Akindes et al. (2012) ont indiqué que ces pressions agricoles, essentiellement le fait d'autochtones Guéré, étaient pour une bonne part le résultat de la confusion créée par la délimitation erronée de 1974, communément appelée limite Goué.

Pendant la période des conflits, le PNMP a connu une forte déforestation. Environ 28,03\% $\left(80,28 \mathrm{~km}^{2}\right)$ de la surface forestière initiale ont été convertis en non-forêts $(22,9 \%)$ et en sols nus et affleurements rocheux $(5,13 \%)$. Ce parc, à l'instar de la forêt classée du Haut-Sassandra (FCHS) où plus de la moitié du paysage $\left(520 \mathrm{~km}^{2}\right)$ a été convertie en non-forêts (Bamba et al., 2018), est un cas typique de déforestation des aires protégées en Côte d'Ivoire durant la période des conflits armés. Les résultats de l'étude d'Ousmane et al. (2018) ont montré que les conflits ont favorisé la prolifération de la culture illégale de cacao dans ce parc, tout comme ceux de Assalé et al. (2016) dans la FCHS. L'infiltration massive du parc par les paysans pendant les conflits s'expliquerait par la fragilisation de son dispositif de surveillance et de gestion (OIPR, 2017 ; Ousmane et al., 2018). Les travaux de plusieurs auteurs corroborent cet état de fait à travers le monde (Mc Neely, 2003 ; Allnutt et al., 2013). Par ailleurs, il faut noter que les pertes massives de superficies forestières pendant la période des conflits ne sont pas seulement le fait des pressions agricoles. L'exploitation forestière en est l'une des causes sousjacentes (Ousmane et al., 2018). Les résultats des travaux d'Akindes et al. (2012) indiquent que cette activité illégale aurait été perpétrée entre 2002-2007 et 2011-2013 par des scieries localisées dans la zone de Duékoué, Daloa et San Pedro, sous la supervision des bandes armées.

La plus grande augmentation de la superficie des sols nus et affleurements rocheux enregistrée à cette période serait due aux activités de défrichement des forêts et de création de plusieurs campements ou villages à l'intérieur du parc. 
Au cours de la période post-conflits allant de 2011 à 2016, le PNMP a enregistré la plus grande perte de superficie forestière $(34,02 \%)$. Cette perte n'est pas très élevée comparée à celle observée durant la période des conflits (28,03\%). Cependant, le taux de déforestation annuel au cours de cette période post-conflits $(14,41 \%)$ est largement supérieur à celui de la période des conflits (3,92\%). Cette situation traduit clairement une intensification du phénomène de déforestation à cette période, comme l'ont indiqué les résultats des travaux de Barima et al. (2016) dans la FCHS. Robert (2015) a fait ce même constat dans son étude sur la dynamique paysagère des forêts d'A Lưới après la guerre du Viêt Nam. L'intensification de la déforestation dans le PNMP à cette période serait due à l'occupation et à l'exploitation accélérée des ressources forestières par la bande armée contrôlant jusque-là le parc et les populations paysannes infiltrées. En effet, entre 2011 et 2013, le PNMP était toujours entièrement contrôlé par la bande armée qui a d'ailleurs entretenu les différentes formes d'exploitation afin d'en tirer un maximum de profits. En 2013, en dépit de la neutralisation de cette bande, le parc est resté toujours occupé par les populations paysannes qui entretenaient les plantations existantes et s'adonnaient à de nouveaux défrichements.

Malgré sa localisation dans la zone tampon, placée sous le contrôle des forces onusienne et française, le PNMP a subi une forte déforestation. Également, les résultats des travaux de Bamba et al. (2018) ont montré que les formations végétales les plus perturbées durant les conflits en Côte d'Ivoire sont celles situées partiellement ou totalement dans la zone tampon. Cette situation laisse considérer que les forces impartiales mandatées par l'ONU pour la surveillance de cette zone ne se sont pas impliquées réellement dans la protection de l'environnement. Cette passivité des forces impartiales vis-à-vis de la question environnementale a été constatée au Congo et dans bien des pays en conflit (Smouts, 2000). Face à cette situation, la création d'une force verte internationale pour la protection des zones importantes de la biodiversité devrait être envisagée (Sand, 1993). De même, le concept de parcs de la paix, initialement lancé pour la création de parcs tels que la chaîne des volcans des Virunga, entre le Rwanda, l'Ouganda et la République démocratique du Congo (Kalpers et Lanjouw, 1998), pourrait être appliqué aux parcs situés dans des zones de guerre. Westing (1992) a souligné qu'il est crucial que les parcs nationaux et les sites de patrimoine mondial deviennent officiellement des zones de non-belligérance en périodes de conflit.

En général, la difficulté d'accès à une zone de guerre, combinée à une absence de définition spatiale et temporelle précise de l'étendue du conflit, rend extrêmement difficile l'évaluation des impacts (Glew et Hudson, 2007). En raison de ces limites, la télédétection se révèle indiscutablement comme l'outil le mieux capable de donner une idée de la manière dont les conflits affectent directement le paysage physique en temps de guerre et conduisent indirectement à des changements dans les activités d'utilisation des sols qui entraînent des modifications dans la couverture terrestre. Les impacts de la guerre sur l'environnement peuvent se ressentir à la fois avant et après un conflit (Machlis et Hanson, 2008). Dans le cadre de la présente étude, la sélection des séries chronologiques d'images satellitaires couvrant les périodes d'avant, pendant et après les conflits est donc une approche rigoureuse afin de déterminer les changements résultant des effets de la guerre sur le Parc national du Mont Péko.

De plus, la technique de détection de changement à base de pixels post-classification adoptée est très adaptée à de telles études basées sur l'usage des images satellitaires multi-temporelles issues de capteurs différents. Cette technique minimise les différences entre les capteurs, l'atmosphère et l'environnement, ainsi que les problèmes liés à l'utilisation d'images multi-capteurs (Mishra et al., 2017). En effet, les images de deux dates sont classées séparément, ce qui minimise le problème de normalisation des différences atmosphériques et de capteurs. La limite de cette technique est que la fiabilité des données produites dépend entièrement de la précision de classification de chaque image.

Concernant la méthode de classification supervisée adoptée dans la présente étude, des points d'entraînement et de validation de bonne précision spatio-temporelle étaient nécessaires pour réaliser des classifications précises. Cependant, l'acquisition de tels jeux de données, en particulier pour la classification des images anciennes (2011, 2002 et 1988), a été une tâche difficile au fur à mesure que l'on se projetait dans le passé. Toutefois, la stratégie de prospection approfondie de la zone d'étude, suivie du traitement de l'image la plus récente, a permis de surmonter cette difficulté. En effet, la connaissance du terrain a garanti la collection des points de contrôle escomptés avec précision dans les images archives haute résolution de Google Earth.

En référence à l'échelle de Landis et Koch (1977), on peut conclure que les résultats de cette étude sont statistiquement acceptables. Il convient cependant de noter que les grandes précisions cartographiques obtenues peuvent aussi dépendre du nombre réduit de classes utilisées (Mama et al., 2013).

\section{Conclusion}

Les pressions anthropiques exercées sur le Parc national du Mont Péko entre 1988 et 2016 ont énormément impacté la dynamique de sa végétation. Cette étude a permis, grâce à l'usage combiné de la télédétection et des systèmes d'information géographique, d'analyser les changements d'occupation des sols intervenus dans cette aire protégée, suivant les périodes d'avant, pendant et après les conflits armés en Côte d'Ivoire.

Il ressort de l'analyse des différentes périodes étudiées une forte réduction des surfaces forestières au profit des exploitations agricoles à base de cacaoyer (non-forêts) durant les conflits, qui s'est accentuée pendant la période post-conflit. Cette déforestation s'est opérée sous l'égide d'une bande armée qui en tirait économiquement profit. L'accélération de la déforestation après l'arrêt des conflits est liée à la volonté de maximiser les profits par tous les acteurs de la chaîne du processus de l'exploitation illégale du parc durant la période post-conflit. Le taux de perte 
globale de la forêt est passé de 5,65 \% avant les conflits (1988-2002) à 28,03 \% pendant les conflits (2002-2011) et à 34,02 \% après les conflits (2011-2016). Ainsi, l'infiltration massive du parc pendant les années de conflit a exacerbé les pressions existantes sur les ressources forestières.

En outre, depuis 2013, dans le cadre d'un vaste programme de sécurisation des aires protégées en Côte d'Ivoire, le gouvernement ivoirien a entrepris de nombreuses actions sur le Parc national du Mont Péko en vue de sa réhabilitation. Ces actions ayant conduit à l'apurement total du parc en 2016 méritent d'être poursuivies par l'instauration d'une surveillance constante et un aménagement des zones converties en plantation de cacao afin d'accélérer la régénération.

\section{Remerciements}

Les auteurs remercient le Programme d'appui stratégique à la recherche scientifique en Côte d'Ivoire (PASRES) pour le financement de cette étude. Également, les auteurs remercient le Centre d'excellence sur les changements climatiques, la biodiversité et l'agriculture durable (CEA-CCBAD) pour avoir offert un cadre de travail propice et une allocation de recherche complémentaire au doctorant Sidibé Ousmane.

\section{Références bibliographiques}

Akindes F., Kouamé W. K., Sédia G., Sezan J., 2012. Réalisation d'une étude sur l'état de la biodiversité des parcs nationaux et réserves de Côte d'Ivoire. Aspects socio-économiques. Rapport provisoire. Abidjan, Côte d'Ivoire, ministère de l'Environnement et du Développement durable, 103 p.

Alix-Garcia J., Anne B., David S., 2013. The landscape of conflict: forced migration and land use in Darfur. Journal of Economic Geography, 13 (4): 589-617. https://doi. org/10.1093/jeg/lbs044

Alix-Garcia J., Saah D., 2010. The effect of refugee inflows on host communities: evidence from Tanzania. World Bank Economic Review, 24 (1): 148-170. https://doi.org/10.1093/ wber/lhp014

Allnutt T., Asner G., Golden C., Powell G., 2013. Mapping recent deforestation and forest disturbance in northeastern Madagascar. Tropical Conservation Science, 6: 1-15. https://doi.org/10.1177/194008291300600101

Alvarez M., 2003. Forests in the time of violence: conservation implications of the Colombian war. Journal of Sustainable Forestry, 16 (3-4): 47-68. https://doi.org/10.1300/ j091v16n03 03

Assalé A. A. Y., Barima Y. S. S., Kouakou K. A., Kouakou A. T. M., Bogaert J., 2016. Agents de dégradation d'une aire protégée après une décennie de conflits en Côte d'Ivoire : cas de la forêt classée du Haut-Sassandra. International Journal of Innovation and Scientific Research, 22 (1) : 123-133.
Avenard J.-M., 1971. Aspects de la géomorphologie. In : Avenard J.-M., Edlin M., Girard G., Sircoulon J., Touchebeuf P., Guillaumet J.-L., Adjanohoun E. (éds). Le milieu naturel de la Côte d'Ivoire. Paris, France, Orstom, 7-71.

Bamba I., Barima Y. S. S., Sangne Y. C., Andrieu J., AssiKaudjhis J. P., 2018. Partition du territoire et dynamique des végétations pendant la période de conflit en Côte d'Ivoire. Tropicultura, 36 (2) : 141-154.

Barima Y. S. S., Kouakou A. T. M., Bamba I., Sangne Y. C., Godron M., Andrieu J., et al., 2016. Cocoa crops are destroying the forest reserves of the classified forest of HautSassandra (Ivory Coast). Global Ecology and Conservation, 8: 85-98. https://doi.org/10.1016/i.gecco.2016.08.009

CEPF, 2000. Écosystème forestier de haute Guinée dans la zone prioritaire de biodiversité de Guinée (Afrique de l'Ouest). Version finale. Arlington, USA, Conservation International, $56 \mathrm{p}$.

Dudley J. P., Ginsberg J. R., Plumptre A. J., Hart J. A., Campos L. C., 2002. Effects of war and civil strife on wildlife and wildlife habitats. Conservation Biology, 16 (2): 319-329. https://doi.org/10.1046/i.1523-1739.2002.00306.x

Eldin M., 1971. Le climat. In : Avenard J.-M., Edlin M., Girard G., Sircoulon J., Touchebeuf P., Guillaumet J.-L., Adjanohoun E. (éds). Le milieu naturel de la Côte d'Ivoire. Paris, France, Orstom, 73-108.

FAO, 1990. Forest resources assessment. Survey of tropical forest cover and study of change processes. Roma, Italia, FAO, Forestry Paper, 130, 152 p.

Girard M.-C., Girard C. M., 1999. Traitement des données de télédétection. Paris, France, Dunod, 529 p.

Glew L., Hudson M. D., 2007. Gorillas in the midst: the impact of armed conflict on the conservation of protected areas in sub-Saharan Africa. Oryx, 41 (2): 140-150.

Goné Bi Z. B., Kouamé D., Koné I., Adou Yao C. Y., 2013. Diversité végétale et valeur de conservation pour la Biodiversité du Parc National du Mont Péko, une aire protégée, menacée de disparition en Côte d'Ivoire. Journal of Applied Biosciences, 71: 5753-5762. https://doi.org/10.4314/jab.v71i1.98820

Gorsevski V., Geores M., Kasischke E., 2013. Human dimensions of land use and land cover change related to civil unrest in the Imatong Mountains of South Sudan. Applied Geography, 38: 64-75. https://doi.org/10.1016/j. apgeog.2012.11.019

Kalpers J., Lanjouw A., 1998. Potentialités pour la création d'un Parc pour la Paix dans la région des volcans Virunga. Cahiers d'Éthologie, 18: 187-206.

Kim K. C., 1997. Preserving biodiversity in Korea's demilitarized zone. Science, 278 (5336): 242-243. https://doi. org/10.1126/science.278.5336.242

Kouakou A. T. M., Barima Y. S. S., Kouakou K. A., Kouamé N. F., Bogaert J., Kouadio J. Y., 2015. Forest dynamics in the North of the classified forest of Haut-Sassandra during the period of armed conflicts in Ivory Coast. American Journal of Life Sciences, 3 (5): 375-382. https://doi.org/10.11648/i. ajls.20150305.17 
Landis J. R., Koch G. G., 1977. The measurement of observer agreement for categorical data. Biometrics, 33: 159-74. https://doi.org/10.2307/2529310

Lauginie F., 2007. Conservation de la nature et aires protégées en Côte d'Ivoire. Abidjan, Côte d'Ivoire, CEDA/NEI, 668 p.

Machlis G. E., Hanson T., 2008. Warfare ecology. BioScience, 58: 729-736. https://doi.org/10.1641/b580809

Mama A., Sinsin B., De Cannière C., Bogaert J., 2013. Anthropisation et dynamique des paysages en zone soudanienne au nord du Bénin. Tropicultura, 31 (1): 78-8.

Mc Neely J. A., 2003. Conserving forest biodiversity in times of violent conflict. Oryx, 37 (2): 142-152. https://doi. org/10.1017/s0030605303000334

Mishra S., Shrivastava P., Dhurvey P., 2017. Change detection techniques in remote sensing: A review. International Journal of Wireless and Mobile Communication for Industrial Systems, 4 (1): 1-8.

Nackoney J., Molinario G., Potapov P., Turubanova S., Hansen M. C., Furuichi T., 2014. Impacts of civil conflict on primary forest habitat in northern Democratic Republic of the Congo, 1990-2010. Biological Conservation, 170: 321-328. https://doi.org/10.1016/i.biocon.2013.12.033

OIPR, 2017. Plan d'aménagement du Parc national du Mont Péko. Version provisoire. Duékoué, Côte d'Ivoire, OIPR, 35 p. Ordway E. M., 2015. Political shifts and changing forests: Effects of armed conflict on forest conservation in Rwanda. Global Ecology and Conservation, 3: 448-460. https://doi. org/10.1016/j.gecco.2015.01.013

Ousmane S., Kouassi K. H., Zadou D. A., Kouame D., Karidia T., 2018. Dynamics of human pressures on the Mont Péko National Park (West-Côte d'Ivoire). European Journal of Scientific Research, 14 (11): 109-124. https://doi.org/10.19044/ esj.2018.v14n11p109

Pettersson T., Eck K., 2018. Organized Violence, 1989-2017. Journal of Peace Research, 55 (4): 535-547. https://doi. org/10.1177/0022343318784101

PNUE, 2015. Côte d'Ivoire : évaluation environnementale post-conflit. Nairobi, Kenya, PNUE, 160 p.

Pontius Jr. R. G., 2000. Quantification error versus location error in comparison of categorical maps. Photogrammetric Engineering and Remote Sensing, 66 (8): 1011-1016.

Puyravaud J.-P., 2003. Standardizing the calculation of the annual rate of deforestation. Forest Ecology and Management, 177: 593-596. https://doi.org/10.1016/s03781127(02)00335-3

Robert A. C., 2015. Les impacts de la guerre du Việt Nam sur les forêts d'A Lưới. Vertigo, 15 (1): 1-24. https://doi. org/10.4000/vertigo.16105

Sand P., 1993. International environmental law after Rio. European Journal of International Law, 4 : 377-389.

Schlaepfer R., 2002. Analyse de la dynamique du paysage. Fiche d'enseignement 4.2. Laboratoire de gestion des écosystèmes, École polytechnique de Lausanne, Suisse, 10 p.
Smouts M.-C., 2000. Un monde sans bois ni lois. La déforestation des pays tropicaux. Critique Internationale, 9 (1) : 131-146. https://doi.org/10.3406/criti.2000.1630

UICN, 1996. L'Atlas pour la conservation des forêts tropicales d’Afrique. Paris, France, Éd. Jean-Pierre de Monza, $310 \mathrm{p}$.

Westing A., 1992. Protected natural areas and the military. Environmental Conservation, 19 (4): 343-348. https://doi. org/10.1017/s0376892900031477

\begin{tabular}{|c|c|}
\hline \multicolumn{2}{|c|}{ Ousmane et al. - Contribution des auteurs } \\
\hline Rôle du contributeur & Noms des auteurs \\
\hline Conceptualisation & $\begin{array}{l}\text { S. Ousmane, H. N'da Dibi, } \\
\text { K. H. Kouassi }\end{array}$ \\
\hline Gestion des données & S. Ousmane \\
\hline Analyse formelle & S. Ousmane \\
\hline $\begin{array}{l}\text { Acquisition du } \\
\text { financement }\end{array}$ & S. Ousmane \\
\hline $\begin{array}{l}\text { Enquête et } \\
\text { investigation }\end{array}$ & S. Ousmane \\
\hline Méthodologie & $\begin{array}{l}\text { S. Ousmane, H. N'da Dibi, } \\
\text { K. H. Kouassi, K. E. Kouassi }\end{array}$ \\
\hline Gestion de projet & $\begin{array}{l}\text { S. Ousmane, K. H. Kouassi, } \\
\text { K. E. Kouassi }\end{array}$ \\
\hline Ressources & S. Ousmane \\
\hline Logiciels & S. Ousmane \\
\hline Supervision & K. H. Kouassi, K. E. Kouassi \\
\hline Validation & $\begin{array}{l}\text { H. N'da Dibi, K. H. Kouassi, } \\
\text { K. E. Kouassi, K. Ouattara }\end{array}$ \\
\hline Visualisation & S. Ousmane \\
\hline $\begin{array}{l}\text { Écriture - Préparation } \\
\text { de l'ébauche originale }\end{array}$ & S. Ousmane \\
\hline $\begin{array}{l}\text { Écriture - Révision et } \\
\text { édition }\end{array}$ & $\begin{array}{l}\text { H. N'da Dibi, K. H. Kouassi, } \\
\text { K. E. Kouassi, K. Ouattara }\end{array}$ \\
\hline
\end{tabular}

Bois et Forêts des Tropiques - Revue scientifique du Cirad
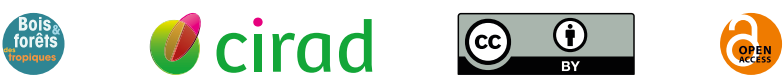

Cirad - Campus international de Baillarguet, 34398 Montpellier Cedex 5, France - Contact : bft@cirad.fr - ISSN : L-0006-579X 